\title{
The Crystal Structure, Orientation Relationships and Interfaces of the Nanoscale Oxides in Nanostructured Ferritic Alloys
}

\author{
Yuan $\mathrm{Wu}^{* 1}$, Jim Ciston$*^{2}$, Stephan $\mathrm{Kräemer}^{1}$, Nathan Bailey ${ }^{3}$, G. Robert Odette ${ }^{1}$, \\ Peter Hosemann ${ }^{3}$ \\ 1. Materials Department, University of California Santa Barbara, CA 93106-5050 \\ 2. National Center for Electron Microscopy, Molecular Foundry, Lawrence Berkeley National \\ Laboratory, 1 Cyclotron Road, Berkeley, CA 94720 \\ 3. Department of Nuclear Engineering, University of California at Berkeley, 4155 Etcheverry Hall, MC \\ 1730, Berkeley, CA 94720
}

\begin{abstract}
The capability of nanostructured ferritic alloys (NFAs) to manage high levels of transmutation product helium will help resolve one of the grand challenges to transforming the promise of C-free fusion energy into a reality. NFAs are dispersion strengthened by an ultrahigh density of Y-Ti-O nano-oxides (NOs), which result in both high strength and temperature limits, as well as unique irradiation tolerance. Here, aberration-corrected high-resolution transmission electron microscopy was used to characterize the NOs in four NFA conditions, including following severe deformation and extreme neutron radiation exposure. Fast Fourier Transform analysis of focal series images revealed the NO crystal structure, including the smallest at $<2 \mathrm{~nm}$ in diameter, to be $\mathrm{Y}_{2} \mathrm{Ti}_{2} \mathrm{O}_{7}$ pyrochlore in all cases, consistent with both exit wave analysis and scanning transmission Z-contrast imaging of the atomic columns in a larger feature. The faceted NOs exhibit a quasi-epitaxial orientation relationship with the ferrite matrix: $[110]_{\mathrm{YTO}} \|[100]_{\mathrm{Fe}}$ and $[001]_{\mathrm{YTO}} \|[010]_{\mathrm{Fe}}$, forming a $5 \times 7$ near coincidence site interface. The NOs also exhibit size-dependent strains in both the oxide and matrix ferrite phases.
\end{abstract}

Keywords: ODS steels, nanoscale oxides, high-resolution electron microscopy, scanning transmission electron microscopy, nanostructured ferritic alloys. 


\section{Introduction}

Fusion reactors will require advanced materials with tolerance to intense high-energy neutron fluxes that generate helium concentrations reaching thousands of atomic parts per million and hundreds of atomic displacements per atom over the operating lifetime. The neutron damage creates effects like void swelling, embrittlement and irradiation creep [13]. Managing helium is a grand challenge for turning the promise of $\mathrm{C}$-free fusion power into a reality. Nanostructured ferritic alloys (NFAs) are dispersion strengthened typically by an ultrahigh number $\left(\approx 5 \times 10^{23} / \mathrm{m}^{3}\right)$ of highly stable nano-oxides (NOs) averaging $\approx 2.5$ $\mathrm{nm}$ in diameter, with volume fractions of $\approx 0.75 \%[1-3]$. NOs pin dislocations, help to self-heal vacancy and self-interstitial damage; and, most significantly, trap otherwise highly damaging helium in harmless nm-scale interface bubbles [1-3]. Thus understanding of NOs is essential to developing, qualifying and optimizing NFAs.

NFAs are often described by their base $\approx 14 \mathrm{Cr}$ content and microalloying solutes $\mathrm{Y}$, $\mathrm{W}$ and $\mathrm{Ti}$, as a 14YWT nano-dispersion strengthened developmental variant of oxide dispersion strengthened steels. Typical NFA compositions are (wt\%): 14Cr, 1-3W (or 0.75-1.5Mo), 0.2-1Ti, 0.15-0.3Y, 0.1O, bal. Fe. The Y-Ti-O atoms are dissolved by a combination gas atomization of powders and mechanical alloying by ball milling $[1,4]$. The NOs precipitate during hot powder consolidation $[1,2,4]$.

However, the chemistry and crystal structure of NOs are still debated [1, 2]. This may partly derive from the different character of NOs found by various techniques: atom probe tomography (APT), transmission electron microscopy (TEM), small angle neutron 
and x-ray scattering (SANS and SAXS) and x-ray diffraction (XRD). Further, with the exception of a reference NFA MA957 [5], most previous studies involved various NFA heats, with somewhat different chemistries and processing paths. A partial summary of previous TEM studies includes: a) Brandes et al. concluded that the smallest NOs are amorphous [6]; b) Hirata et al. reported coherent Ti-rich defective $\mathrm{NaCl}$ structures [7]; c) London et al. found that $\mathrm{NOs}>2 \mathrm{~nm}$ are $\mathrm{Y}_{2} \mathrm{Ti}_{2} \mathrm{O}_{7}$ or $\mathrm{Y}_{2} \mathrm{TiO}_{5}$ [8]; d) Ribis et al. and Dawson et al. reported impressive atomic resolution images of $<5 \mathrm{~nm} \mathrm{Y}_{2} \mathrm{Ti}_{2} \mathrm{O}_{7}-$ both found cube-on-cube and cube-on-edge orientations relationships [9-11]; e) Bhattacharya et al. identified $\mathrm{Y}_{2} \mathrm{Ti}_{2} \mathrm{O}_{7}$ NOs and larger $\mathrm{Y}_{2} \mathrm{TiO}_{5}$-like precipitates [12]; f) detailed measurements by $\mathrm{Wu}$ et al., including selected area diffraction and energy dispersive $\mathrm{x}$ ray spectroscopy on large numbers of small $(<5 \mathrm{~nm}$ and peaking at $2.75 \pm 0.5 \mathrm{~nm}$ ) aggregated NOs extracted from MA957 on a C-foil, showed that the nano-scale features are primarily $\mathrm{Y}_{2} \mathrm{Ti}_{2} \mathrm{O}_{7}$; however, this study noted that there could be differences between embedded and extracted NOs [5]; g) most other TEM and XRD studies find $\mathrm{Y}_{2} \mathrm{Ti}_{2} \mathrm{O}_{7}$ and $\mathrm{Y}_{2} \mathrm{TiO}_{5}$ as discussed in $[2,5]$.

SANS measurements are also reasonably consistent with $\mathrm{Y}_{2} \mathrm{Ti}_{2} \mathrm{O}_{7}$ and $\mathrm{Y}_{2} \mathrm{TiO}_{5}$ [13]. However, previous APT studies have suggested that NOs are non-stoichiometric transition phases, highly enriched in $\mathrm{Ti}, \mathrm{Cr}$ and $\mathrm{Fe}$ [13-19]. The APT Y/Ti ratios have been reported to be much less than 1 ranging from $\approx 0.1$ to 0.5 , and most typically from $\approx$ 0.2 to 0.3 . These same APT studies almost always also show non-stoichiometric O deficiencies, with $\mathrm{O} /(\mathrm{Y}+\mathrm{Ti})$ ratios of $\approx 1$, or even less, in some cases. Note, APT suffers from a number of artifacts that can confound NO composition measurements, including trajectory aberrations, surface diffusion, multiple ion evaporation loss, solute ion pre- 
emission between voltage or laser pulses and chromatic aberrations [20]. For example it is now generally accepted that the high matrix $\mathrm{Fe}$, and to some extent $\mathrm{Cr}$, contents in the NOs are due to trajectory aberrations caused by local dimpling caused by corresponding caused by more rapid evaporation of the NO atoms than the surrounding matrix. This artifact is clearly reflected in high local atom densities in the NO region that are up to more than 3 times what is physical. The $\mathrm{O}$ deficiency is at least partly caused by surface diffusion of $\mathrm{O}$ atoms during tip evaporation [21-23]. The low Y/Ti ratio may be due to a thin $\mathrm{Ti}$, or $\mathrm{Ti}$ and $\mathrm{Cr}$, enriched shell surrounding the $\mathrm{Y}_{2} \mathrm{Ti}_{2} \mathrm{O}_{7} \mathrm{NO}$, which is projected into the core at a dimple due to trajectory aberrations; this is often manifested as a $\mathrm{Ti}, \mathrm{Cr}$ and O enriched tail artificially placed at the bottom of the reconstructed feature [18,21-22]. Other explanations of the low $\mathrm{Y} / \mathrm{Ti}$ and $\mathrm{O} / \mathrm{M}$ ratio have been offered such as uncorrected charge to mass ratio peak overlaps and loss of ions due to unresolved multiple or delayed hits on the detector $[15,24]$. Finally, perhaps it is most notable that APT measurements by both Williams and our own studies on bulk stoichiometric $\mathrm{Y}_{2} \mathrm{Ti}_{2} \mathrm{O}_{7}$ also show low $\mathrm{O} / \mathrm{M}$ and $\mathrm{Y} / \mathrm{Ti}$ ratios, yielding a composition of $18 \% \mathrm{Y}, 33 \% \mathrm{Ti}$ and $49 \% \mathrm{O}$, prior to corrections for mass spectrum peak overlaps and incorrect ion identification within multiple detector hits [25]. In summary, clearly the NO structures and compositions are becoming increasingly well understood.

The results reported here provide both further confirmatory evidence, and a number of completely new results, that address the following issues:

- While there is an emerging consensus that the NO are predominantly pyrochlore $\mathrm{Y}_{2} \mathrm{Ti}_{2} \mathrm{O}_{7}$, details such as the precise stoichiometry and defect structures are still being debated, 
- The effects severe deformation and extreme neutron irradiation exposures on NOs remain important questions?

- Detailed indexing of the structures of the NOs below $\approx 2 \mathrm{~nm}$ is an unmet challenge?

- The reported NO orientation relationships with the ferrite matrix vary and need further study?

- The detailed structure of the NO-ferrite atomic scale interface has bot been characterized at the atomic scale.?

- The strains in the NO and surrounding ferrite matrix and how they depend on NO size have not been quantified?

While we certainly do not claim that the detailed new results reported here provide a final, or comprehensive, resolution of all of these difficult issues, they are arguably an important step in that direction.

Preliminary results were previously summarized for one NFA [26]. Here we study four NFA conditions. Focal-series aberration corrected high resolution TEM (HRTEM) was used to characterize NO crystal structures, interfaces and orientation relationships (ORs) for larger NOs. We compare these high fidelity results to smaller NOs that are much more difficult to individually index.

\section{Experimental}

\subsection{Materials}

TEM studies were carried out on three conditions of MA957 and a similar NFA 14YWT heat recently developed in the DOE Fuel Cycle Research and Development Program called FCRD NFA-1. The variants of MA957 included as-extruded (AE), 
friction stirred weld and annealed (FSWA), and irradiated (IR) conditions. The AE MA957 was obtained from Pacific Northwest National Laboratory in the form of extruded 25-mm-diameter rods [27]. The FSW was prepared by Edison Welding Institute in collaboration with UCSB, by joining two MA957 AE coupons, followed by post-weld annealing at UCSB at $1150^{\circ} \mathrm{C}$ for $1 \mathrm{~h}[28,29]$; annealing was intended to relax residual stresses and to recover any potential damage to the NOs that might be caused by severe plastic deformation. The IR condition is another MA957 heat irradiated to 109 dpa at $412^{\circ} \mathrm{C}$ in the Fast Flux Test Reactor [30]. FCRD NFA-1 is a best practice heat of 14YWT in the form of an extruded and cross-rolled plate [31].

Various combinations of APT, SANS, TEM and mechanical property measurements have been used to extensively characterize these four NFA conditions [5, 12, 13, 27-31]. Table 1 summarizes the size and number density measurements using TEM, APT, and SANS. In general the small NF size and high number density are remarkably similar in all three MA957 conditions, while the newer 14YWT also contains a similar population of NOs. Compared to some other NFA alloys both MA957 and FCRD-NFA1 contain a nearly homogeneous distributions of NOs, except at APT length scales where the number density can vary modestly from tip to tip. Typical size distribution of NOs can be found in Ref. 5.

\subsection{TEM characterization}

Electron transparent TEM specimens were prepared by either the focused ion beam (FIB) lift-out technique, using FEI Helios and Quanta microscopes, or by wedge polishing, followed by ion-milling, both at successively lower energies down to $2 \mathrm{kV}$ to minimize the gallium and argon ion damage. Most of the HRTEM images were recorded 
using the TEAM 0.5 FEI-Titan-class microscope with aberration correction to third order in both TEM and STEM modes [32]. HRTEM data was collected at $300 \mathrm{kV}$ using monochromation to $0.1 \mathrm{eV}$ with the corrector tuned to optimize bright atom contrast (C3 $=-6 \mathrm{um}, \mathrm{C} 5=2.5 \mathrm{~mm}$ ) and recorded on a $2048 \times 2048$ pixel CCD camera. Focal series were recorded with a defocus step of $-1.72 \mathrm{~nm}$ ranging from $-34.4 \mathrm{~nm}$ underfocus to 34.4 $\mathrm{nm}$ overfocus. Exit wave reconstruction was performed using the Gershberg-Saxton algorithm [33] as implemented in the MacTempasX software [34]. Exit wave reconstruction is a holographic technique that can provide both the amplitude and phase of the electron wave after passing through the sample. Once the complex wave is known, all residual aberrations of the microscope can be corrected by software [35], improving the interpretability of the HRTEM data. Furthermore, at the limit of the weak phase object approximation, the exit wave phase is directly related to the projected potential of the object. MacTempasX was also used to perform multislice HRTEM simulations using the experimental parameters and a cutoff frequency of $10 / \mathrm{nm}$. Atomic coordinates of the Fe- $\mathrm{Y}_{2} \mathrm{Ti}_{2} \mathrm{O}_{7}-\mathrm{Fe}$ slab are provided in Supplemental Materials. TEAM 0.5 was also used in STEM mode to record high angle annular dark field (HAADF) images with a $17.1 \mathrm{mrad}$ convergence semi-angle and a collection semi-angle ranging from 75-150 mrad. Finally, electron energy loss spectroscopy (EELS) was used to characterize Ti-profiles for a limited number of NOs. The HRTEM images and exit waves were analyzed by FFT power spectrum lattice indexing. Individual FFT were first calculated and then collectively averaged using MATLAB. The lattice spacings and inter-planar angles were measured using Image $\mathrm{J}$. 
As is typical of HRTEM studies, only limited numbers of NOs were examined in great detail with the full exit wave analysis and STEM methods described above. These numbers are naturally limited by the presence of an optimal matrix zone axis coupled with limitations on effects of the size and defect characteristics of NOs; time limits at a user facility balanced against needs for other measurements were also a factor. Thus only two individual nano-scale features were examined in HRTEM through focus detail, while nine others were indexed based on more usual HRTEM FFT methods. Thus other variants of OR relationships, like cube-on-cube that have been reported by others $[9,11]$, may well be present that escaped direct observation in this study. However, there is also no evidence that our observations are not representative of an important fraction of NOs in NFAs. This argument is bolstered by the novel application of the wide area diffraction characterization of the smaller oxides that observed collective reflections from many NOs, as discussed below.

\section{Results}

The microstructures of the 4 NFA conditions were characterized by aberration corrected TEM: MA 957 in as-extruded (AE), friction stir welded and annealed (FSWA) and irradiated (IR) conditions; and a new developmental heat of 14YWT (NFA-1). Note that the FSWA and IR conditions represent extreme conditions of deformation and neutron irradiation exposure, respectively. Figure 1a is a bright field TEM image of the MA957 FSWA condition, showing the NO features. The NOs typically have elongated cuboidal shapes with sizes widely ranging from $\approx 1$ to $12 \mathrm{~nm}$. The left side of Figure $1 \mathrm{~b}$ shows three HRTEM images of the same NO, as examples of a larger series representing (i) under-focused; (ii) close-to-focused; and, (iii) over-focused conditions. The NO is 
sharply faceted and the Moire patterns indicate that it is crystalline. The right side of the Figure $1 \mathrm{~b}$ shows the associated FFT for each of these focal condition examples. The overlay of stronger and weaker lattice spots represent reflections of the ferrite matrix and the NO, respectively. The weak NO reflections vary in amplitude with the focal condition and, in some cases, even disappear. This is a result of the strong image modulations introduced by the contrast transfer function of the TEM objective lens.

Taking the average over all fast Fourier transforms (FFTs) in a focal series minimizes contrast transfer function effects and provides a higher signal-to-noise ratio. The corresponding averaged FFT is shown in Figure 1c. The strong reflections (with a red spot in the middle) are the $\langle 100\rangle$ zone-axis pattern of the bcc ferrite matrix. The primary reflections from the NOs, marked by white circles, are consistent with $\mathrm{Y}_{2} \mathrm{Ti}_{2} \mathrm{O}_{7}$ pyrochlore crystals with a $\langle 110>$-zone axis. Detailed analysis shows that all the other spots arise from multiple scattering.

The in-focus electron wave phase obtained from exit-wave reconstruction (EWR) in Figure 2 takes into account the transfer function modulations, and provides a more interpretable representation of the projected crystal structure. Figure 2a displays the phase of the reconstructed HRTEM exit wave, with white atom contrast, while Figure $2 b$ shows the corresponding HAADF STEM image. In both cases a periodic image pattern is observed in the central region of the large NO, reflecting the overlap of the ferrite matrix and the $\mathrm{Y}_{2} \mathrm{Ti}_{2} \mathrm{O}_{7}$ pyrochlore crystal structures. The in-focus phase of the exit wave and HAADF STEM images are both interpreted as projected atomic columns, as supported by corresponding HRTEM simulations in Supplementary Figure S1. Corresponding overlays of an ideal $\mathrm{Y}_{2} \mathrm{Ti}_{2} \mathrm{O}_{7}$ pyrochlore atomic model with the observed OR are shown in Figures 
$2 \mathrm{c}$ and $2 \mathrm{~d}$. The dashed lines correspond to a periodic array of $5 \times 7 \mathrm{Fe}$ periodic cells, while the colored balls represent lateral positions of Y (green) and $\mathrm{Ti}$ (blue) atoms relative to the Fe matrix (yellow), based on a visual best fit position adjustment of the image intensities. Note the visible atoms represent columns of pure Y, pure Ti and mixed Y and Ti. The bulk $\mathrm{Y}_{2} \mathrm{Ti}_{2} \mathrm{O}_{7}$ model is very well matched to the EWR and STEM column patterns, when displaced relative to the ferrite matrix, based on simple visual pattern matching. The periodic pattern corresponds to a $5 \times 7$ Fe periodic cell near coincident site lattice.

A smaller $1.8 \times 3.3 \mathrm{~nm}$ rectangular-shaped oxide with pyrocholre structure is shown in Fig. 3a. The corresponding FFT spectra in Fig. 3b, show the the orientation relationship is also cube-on-edge, i.e. $[110]_{\mathrm{Yto}} \|[100]_{\mathrm{Fe}}$ and $[001]_{\mathrm{Yto}} \|[010]_{\mathrm{Fe}}$.

A HAADF STEM image of AE MA957 is shown Figure 4a. The faceted oxides, indicated by the white circles, are significantly smaller in this case, with most of them less than $2 \mathrm{~nm}$. The number density of NOs here is about $2.4 \times 10^{23} / \mathrm{m}^{3}$. Imaging the smallest NOs in HRTEM is very difficult due to reduced atomic mass contrast, the dominance of interfaces, as well as the possibilities of complex chemical oxide terminations, mismatch strains, solute segregation and the presence of both ferrite matrix lattice and oxide defects. The averaged FFT of one somewhat larger NO in the AE condition, shown in Figure 4b, deviates from those in for the FSWA MA957 in Figure 1c, since there are $\{100\}$ indexed spots lying on a line between the major $\{110\}$ type $\mathrm{Fe}$ reflections, which would be forbidden in an ideal bulk crystal. These extra spots may occur both due to a symmetry-breaking sharp truncation of the bcc Fe crystal at the small NOs interface and possible lattice defects. The other reflection spots visible on the line 
between the $\mathrm{Fe}$ reflections are consistent with a $\mathrm{Y}_{2} \mathrm{Ti}_{2} \mathrm{O}_{7}$ pyrochlore crystal orientated along [100] with a [100] $\mathrm{YTO} \|[010]_{\mathrm{Fe}}$ and [011] $]_{\mathrm{YTO}} \|[100]_{\mathrm{Fe}}$ OR, which is one of those found by both Dawson et al.[11] and Ribis et al. [10]. Note that individual NOs were not indexed for the MA957 IR condition. but this will be part of future research.

Structural information was also obtained from the extra spots in the FFT for a larger image area containing a sufficient volume fraction of NOs, even if they could not be indexed singly due to signal to noise limitations. To the best of our knowledge this broad selected area selected diffraction approach has not been reported previously. The collage in Figure 5 is a summary of corresponding power spectra for all the NFA conditions in this study. Each spectrum is based on the average of all the individual images in the focal series. Representative HRTEM images from the source focal series for each panel in Figure 5 are shown in Supplementary Figure S2. For comparison purposes, Figure 1c is repeated in 5a. Figures $5 \mathrm{~b}$ and $5 \mathrm{c}$ are power spectra from two other regions in FSWA MA957. Figure $5 \mathrm{~b}$ is for the NO, which is $1.8 \mathrm{~nm}$ high and $3.3 \mathrm{~nm}$ wide, in Fig.3. The large aspect ratio can be seen as an elongation of the corresponding reflections along the vertical axis. Due to the smaller size of these NOs, multiple scattering is reduced compared to the larger crystal shown in Figure 1a. The Fe region next to the particle contains no discernible features. The corresponding FFT in Figure 5c mainly contains ferrite matrix spots, with only very minimal indications of any other structures in the background.

The broad area power spectra in Figures 5d and e are FFT for the AE and IR MA957 conditions, respectively, and Figure $5 \mathrm{f}$ is for NFA-1. In contrast to the absence of reflections in the area without NOs in Figure 5c, all the FFT for a larger area of the foil 
show the same extra spots characteristic of $\mathrm{Y}_{2} \mathrm{Ti}_{2} \mathrm{O}_{7}$, i.e. weak peaks in between and close to the $\{200\}$-type Fe reflections. Details are shown in Table 2. The spots that are near the Fe peaks are at the same locations that were previously assigned to $\mathrm{Y}_{2} \mathrm{Ti}_{2} \mathrm{O}_{7}$ pyrochlore in Figure 1. Note Ribis et al. also reported pyrochlore structure is stable after irradiation [10]. However, in contrast to the other images, these spots are streak-like broadened, as discussed in the next section.

A careful analysis of NOs in MA957 and 14YWT NFA-1 heat reveals strongly facetted shapes and a well-defined OR to the ferrite matrix. All of the recorded and indexed power spectra are consistent with a cube-on-edge OR as illustrated in Figure 6a with $[110]_{\mathrm{YTO}} \|[100]_{\mathrm{Fe}}$ and $[001]_{\mathrm{YTO}} \|[010]_{\mathrm{Fe}}$ for all of the alloy conditions.

Due to the two-fold symmetry of the $\langle 110\rangle$-zone axis there are three possible variants of overlapping ferrite and $\mathrm{Y}_{2} \mathrm{Ti}_{2} \mathrm{O}_{7}$ pyrochlore diffraction patterns. Figure $6 \mathrm{~b}$ i) shows possible peak locations in the power spectrum assuming an equal mix of three cube-on-edge rotations. Figures $6 \mathrm{~b}$ ii) and iii) correspond to $<110>$-type $\mathrm{Y}_{2} \mathrm{Ti}_{2} \mathrm{O}_{7}$ pyrochlore patterns rotated $90^{\circ}$, while iv) shows a $<100>$-type pattern that is $45^{\circ}$ rotated relative to $[100]_{\mathrm{Fe}}$. Notably, figures $5 \mathrm{~d}$, e and $\mathrm{f}$ show streaking of the corresponding $\mathrm{Y}_{2} \mathrm{Ti}_{2} \mathrm{O}_{7}$ reflections that are consistent with a combination these three rotation variants.

The strong OR between ferrite matrix and $\mathrm{Y}_{2} \mathrm{Ti}_{2} \mathrm{O}_{7}$ pyrochlore is intriguing given the incommensurate bulk unit cells of the two crystals. Nevertheless, it is noted that the overlapped crystal structures with the specified OR show a near CSL between Fe atoms and $\mathrm{Y}$ and Ti cations that spans $5 \times 7$ ferrite periodic cells, as illustrated in Figure 6c. Note, Figure $6 \mathrm{c}$ represents an yet to be published first principles calculation of an embedded NO, provided by Y. Jiang at the South Central University In China, which was inspired 
by the TEM results reported here. The atomic positions, including $\mathrm{O}$, in a 525 atom supercell are fully relaxed from a bulk pyrochlore structure with the near 5x7 CSL interface with the ferrite matrix. The atomic coordinates of the relaxed structure are provided in Supplementary Material. The first principles minimization resulted in only very small movements of the atoms, suggesting that this interface represents a highly stable structure. Details of this very recent modeling research will be the subject of a future publication, including corresponding interface energies, as well as a full oxygen potential and temperature dependent interface phase diagram.

The repeat units in Figure 6c correspond exactly to the observed periodicity seen in Figure $2 \mathrm{~b}$ and the coherent structures are consistent with the EWA. The lattice mismatch is remarkably low across these periodic distances, at only $+0.45 \%$ along the horizontal and $-0.57 \%$ along the vertical directions. The corresponding strains in the fully relaxed DFT model are horizontal/vertical: $1 / 1.05 \%$ in $\mathrm{Fe}$ and $-1.77 /-0.7 \%$ in $\mathrm{Y}_{2} \mathrm{Ti}_{2} \mathrm{O}_{7}$. Due to the symmetry of the OR, the lattice strains along horizontal and the third, out of plane, direction are equivalent.

The interfaces between the NO and ferrite matrix are also remarkably flat, as is especially seen in the FSWA condition shown in Figure 1; note the other alloy conditions also show faceted NOs. The example of the larger particle in the MA957 AE condition in Figure $4 \mathrm{c}$ is most striking. Nevertheless, upon close inspection similar faceting is observed for the smaller particles. Presumably optimally matched interfaces between low-energy planes on both the oxide and iron matrix sides are self-selected. However, the exact shape of the particles cannot be determined from the projection images we have obtained and need to be further characterized by electron tomography, for example. 
Geometric phase analysis (GPA) [36] of the focal series for the MA957 FSWA condition, illustrated in Figure 1b, was used to estimate the NO and ferrite matrix strains. By measuring the lattice distances and comparing to bulk $\mathrm{Y}_{2} \mathrm{Ti}_{2} \mathrm{O}_{7}$ lattice $\mathrm{d}$ spacing, we calculated that the compressive strains in the NOs ranged from 0.8 to $-9.1 \%$, and increased with decreasing feature size, averaging $-0.3 \%$ for $\approx 8$ by $10 \mathrm{~nm}$ and $\approx-6.5 \%$ for $\approx 1.8$ by $3.3 \mathrm{~nm} \mathrm{Y}_{2} \mathrm{Ti}_{2} \mathrm{O}_{7}$ features, respectively. Compressive strains in the larger NO are balanced by similar tensile strains of $\approx 0.5 \%$ in the ferrite matrix as determined by GPA as shown in Supplemental Figure S3. Ti profiles measured by EELS are consistent with the beveled edges of the NOs seen in the HRTEM images, and closely track in the total HAADF signal profiles.

\section{Discussion}

Returning to the initial list of questions we conclude that: a) the NO are predominantly $\mathrm{Y}_{2} \mathrm{Ti}_{2} \mathrm{O}_{7}$ pyrochlore that appear to persist down to small sizes even in the face of severe deformation and extreme irradiation exposure, and in all four alloy conditions; b) the NOs observed here have a predominantly cube-on-edge [110] $]_{\text {YTO }} \|$ $[100]_{\mathrm{Fe}}$ and $[001]_{\mathrm{YTO}} \|[010]_{\mathrm{Fe}}$ OR relationship in all conditions; c) the atomic scale interface in a larger NO can be described as a 5x7 near CSL; and d) size dependent compressive strains average $-0.3 \%$ for larger, and $\approx-6.5 \%$ for smaller, NOs respectively, and in the case of the larger NO, roughly similar tensile strains are observed in the matrix.

A pertinent question is how general are these results? We note that cube-on-cube ORs were previously reported in Refs. [9] and [11], thus the CSL cube-on-edge interface clearly found in this study may not represent all possibilities, which is not surprising. 
With regard to the structure of the smallest oxides, even wide area TEM FFT measurements only sample a small volume of material. Classical techniques for crystal structure analysis in larger volumes, like XRD, have serious limitations when dealing with low volume fractions of nano-scale crystals, with sizes of order of one to several nm. However, new synchrotron based techniques like the partially or not known structures (PONKS) Rietveld Refinement approach to fit the peak broadened background of the XRD spectra [37] has been recently demonstrated for Ni- Mn-Si nano precipitates in reactor pressure vessel steels at the new NSLS II synchrotron light source at Brookhaven National Laboratory [38]. Application of XRD PONKS to NFAs and NOs will lead to further understanding. Indeed, it is notable that our preliminary XRD results on AE MA957 show weak peaks and enhanced background that are consistent with the presence of $\mathrm{Y}_{2} \mathrm{Ti}_{2} \mathrm{O}_{7}$ and $\mathrm{Y}_{2} \mathrm{TiO}_{5}$ NOs, along with much larger $\mathrm{TiO}$ features. These results are also consistent with several previous XRD studies that characterized greater volume fractions of larger or extracted NOs [39-41]. Furthermore, a very recent glancing angle XRD study found only $\mathrm{Y}_{2} \mathrm{Ti}_{2} \mathrm{O}_{7}$ peaks in a MA957-like 14YWT NFA [42]. Finally, a combination of SAXS and SANS was used to identify the NOs in another 14YWT alloy, showing that they are consistent with $\mathrm{Y}_{2} \mathrm{Ti}_{2} \mathrm{O}_{7}$ or $\mathrm{Y}_{2} \mathrm{TiO}_{5}[43]$.

We have focused on the $\mathrm{Y}_{2} \mathrm{Ti}_{2} \mathrm{O}_{7}$ and, in the case of the larger pyrochlore oxide, the detailed atomic structure of its ferrite matrix interface. However, we should emphasize that, as noted above, other oxides are also often present in NFAs, such as $\mathrm{Y}_{2} \mathrm{TiO}_{5}$, and different cube-on-cube ORs have also been reported [9]. Further, while we believe that the HRTEM images suggest that they are similar, we have not resolved the detailed interface structures in the smallest NOs. We also note that in the other limit of $\mu \mathrm{m}$-scale 
bilayers of Fe grown on (100) $\mathrm{Y}_{2} \mathrm{Ti}_{2} \mathrm{O}_{7}$ pyrochlore, the edge-on-cube $\mathrm{OR}$ is accompanied by interface dislocations with a spacing of $1.4 \mathrm{~nm}$, which are not observed in small embedded NOs that do not experience a semi-infinite in-plane strain condition.

While the space allowed here does not permit a detailed discussion, it is useful to emphasize the critical importance of the detailed observations reported here. For example, the presence of interface strains has important implications to the NO interactions with helium. This is especially the case to support modeling efforts to better understand the nature of NOs, their functionality and the corresponding overall performance of NFAs. The first principles result in Figure $6 c$ is an example of the opportunity for close coupling of modeling and experiment. This work also provides "ground truth" to a series of first principles and atomistic studies that have explored: a) initial clustering energetics of Y-Ti-O solutes [44-46]; b) the structure and physical properties of $\mathrm{Y}_{2} \mathrm{Ti}_{2} \mathrm{O}_{7}$ and $\mathrm{Y}_{2} \mathrm{TiO}_{5}$ [47]; c) the corresponding remarkable ability of NO to sequester helium, in internal interstitial sites and subsequently in associated interface bubbles [48,49]; d) the free surface and embedded interface energies of $\mathrm{Y}_{2} \mathrm{Ti}_{2} \mathrm{O}_{7}[50,51]$; and, e) the solubility of $\mathrm{Y}$ both in the matrix and at dislocations [52], that mediates the truly remarkable long-term, high-temperature thermal stability of NOs, as modeled by cluster dynamics methods [27].

\section{Summary and conclusions}

A detailed high resolution TEM study was conducted to answer unresolved questions about the nature of the NOs present in NFAs. The results suggest that even the smallest cubic and cuboidal NOs are predominately $\mathrm{Y}_{2} \mathrm{Ti}_{2} \mathrm{O}_{7}$ pyrochlore in all the 14YWT alloy conditions we studied. The NOs have a cube-on-edge, apparently coherent, interface that 
manifests a well-ordered 5x7 near CSL oxide-bcc ferrite-matrix boundary structure in a larger oxide. The NOs are under compressive stress while the Fe-matrix is under tension. With the exception of APT measurements, the more detailed results reported here are generally in good agreement with the preponderance of corresponding evidence in the literature although other phases and ORs also occur. The results of this study represent an important step in supporting detailed modeling efforts, such as the first principles simulation of the $5 \times 7$ near CSL example in Figure 6c, that will help to develop, optimize and qualify NFA for fusion and advanced fission energy service.

\section{Acknowledgements}

Research at UCSB was supported by the Department of Energy Office of Fusion Energy Sciences (Grant No. DE-FG03-94ER54275) and the Office of Nuclear Energy (Grant No. DE-FC07-07ID14825). Most of the specimen preparation and some of the TEM measurements were carried out in the UCSB Materials Research Laboratory Microstructure and Microanalysis Facility, supported by the Materials Research Science and Engineering Center Program of the National Science Foundation under Award No. DMR05-20415. The authors thank our UCSB colleagues P. Wells for insight on APT issues, Dr. T. Yamamoto for his many general contributions to our research program, T. Stan for helpful discussions of the interfaces, and especially N. Cunningham for adding to the discussion of APT as well as many others in the Odette Group for their assistance in various parts of this research. We also acknowledge helpful discussions regarding XRD with D. Sprouster and L. Ecker at BNL, and D. Morgan L. Barnard at the University of Wisconsin for their outstanding modeling insight and and Y. Jiang at the Central South University in China sharing the DFT results shown in Figure 5. Electron 
microscopy experiments were performed at the NCEM facility of the Molecular Foundry, which is supported by the Office of Science, Basic Energy Sciences of the U.S. Department of Energy under Contract DE-AC02-05CH11231. The authors would like to thank M. Libbee and C. Song for their support on TEM sample preparation and training at the Molecular Foundry. Finally, we acknowledge the assistance of M. Toloczko at PNNL in providing the irradiated MA957 characterized in this study and S. Maloy at LANL and D. Hoelzer at ORNL for their role as UCSB collaborators in developing FCRD NFA-1. 


\section{References}

[1] G. R. Odette, M. J. Alinger, B. D. Wirth, Recent development in irradiationresistant steels, Annu. Rev. Mater. Res. 38 (2008) 471-503.

[2] G. R. Odette, Recent progress in developing and qualifying nanostructured ferritic alloys for advanced fission and fusion applications, JOM 66 (2014) 24272441.

[3] Y. Dai, G. R. Odette, T. Yamamoto, Basic aspects of radiation effects in solids/basic aspects of multi-scale modeling, Comprehensive Nuclear Material, Elsevier, 2012, Vol 1, Chap 6.

[4] S. Ukai, Radiation effects in structural and functional materials for fission and fusion reactors, Comprehensive Nuclear Material, Elsevier, 2012, Vol. 4, Chap 8.

[5] Y. Wu, E. Haney, N. Cunningham, G. R. Odette, Transmission electron microscopy characterization of the nanofeatures in nanostructured ferritic alloy MA957, Acta Mater. 60 (2012) 3456-3468.

[6] M. C. Brandes, L. Kovarik, M. K. Miller, M. J. Mills, Morphology, structure, and chemistry of nanoclusters in a mechanically alloyed nanostructured ferritic steel, J. Mater. Sci. 47 (2012) 3913-3923.

[7] A. Hirata, T. Fujita, Y. R. Wen, J. H. Schneibel, C. T. Liu and M. W. Chen, Atomic structure of nanoclusters in oxide-dispersion-strengthened steels, Nature Mater. 10 (2011) 922-926.

[8] A. London, S. Lozano-Perez, S. Santra, S. Amirthapandian, B. K. Panigrahi, C. S. Sundar, C. R. M. Grovenor, Comparison of atom probe tomography and transmission electron microscopy analysis of oxide dispersion strengthened steels, J. Phys. Conf. Ser. 522 (2014) 012028.

[9] J. Ribis, Y. de Carlan, Interfacial strained structure and orientation relationships of the nanosized oxide particles deduced from elasticity-driven morphology in oxide dispersion strengthened materials, Acta Mater. 60 (2012) 238-252.

[10] J Ribis. Y. de Carlan, A. Legris, Comparison of the neutron and ion irradiation response of nano-oxides in oxide dispersion strengthened materials, J. Mater. Res. 30 (2015) 2210-2221.

[11] K. Dawson, G. J. Tatlock, Characterisation of nanosized oxides in ODM 401 oxide dispersion strengthened steel, J. Nucl. Mater. 444 (2014) 252-260.

[12] D. Bhattacharyya, P. Dickerson, G. R. Odette, S. A. Maloy, A. Misra, M. Nastsi, On the structure and chemistry of complex oxide nanofeatures in nanostructured ferritic alloy U14YWT, Philos. Mag. 92 (2012) 2089-2107.

[13] M. J. Alinger, G. R. Odette, D. T. Hoelzer, On the role of alloy composition and processing parameters in nanocluster formation and dispersion strengthening in nanostructured ferritic alloys, Acta Mater. 57 (2009) 392-406.

[14] M. K. Miller, K. F. Russell, and D. T. Hoelzer, Characterization of precipitates in MA/ODS ferritic alloys, J. Nucl. Mater. 351 (2006) 261-268.

[15] C. A. Williams, P. Univantowicz, N. Baluc, G. D. W. Smith, and E. A. Marquis, The formation and evolution of oxide particles in oxide-dispersion-strengthened ferritic steels during processing, Acta Mater. 61 (2013) 2219-2235.

[16] M. K. Miller, D. T. Hoelzer, E. A. Kenik, and K. F. Russell, Nanometer scale precipitation in ferritic MA/ODS alloy MA957, J. Nucl. Mater. 329-333 (2004) 338-341. 
[17] M. K. Miller, D. T. Hoelzer, E. A. Kenik, and K. F. Russell, Stability of ferritic MA/ODS alloys at high temperatures, Intermetallics 13 (2005) 387-392.

[18] N. Cunningham, Study of the structure, composition, and stability of Y-Ti-O nmscale features in nano-structured ferritic alloys, Ph.D. Dissertation, University of California Santa Barbara. September 2012.

[19] E. A. Marquis and J. M. Hyde, Applications of atom probe tomography to the characterization of solute behaviours, Materials Science and Engineering: R, 69 (2010) 37-62.

[20] E. A. Marquis, Atom probe tomography allied to the analysis of irradiated microstructures, J. Mater. Res. 30 (2015) 1222-1230.

[21] E. A. Marquis, , Core/shell structures of oxygen-rich nanofeatures in oxidedispersion strengthened Fe-Cr alloys, Appl. Phys. Lett., 93 (2008) 181904.

[22] P. Wells, , N. J. Cunningham, and G. R. Odette, , Recent Progress on Understanding and Quantifying Atom Probe Tomography Artifacts for High Evaporation Rate nm-scale Phases in Fe Based Alloys, Fusion Reactor Materials Semiannual Progress Report. DOE-ER-0313/51 (2011) 9-21.

[23] C. A. Williams,. E. A. Marquis, A. Cerezo, and G. D. W. Smith, Nanoscale characterization of ODS-Eurofer 97 steel: An atom-probe tomography study, J.Nucl. Mater. 400 (2010) 37-45.

[24] A. J. London, S. Lozano-Perez, M. P. Moody, S. Amirthapandian, B. K. Panigrahi, C. S. Sundar, and C. R. M. Grovenor, Quantification of oxide particle composition in model oxide dispersion strengthened steel alloys, Ultramicroscopy( )159, , 360-367.

[25] C. Williams, Atomic scale characterization of oxide dispersion strengthened steels for fusion applications, $\mathrm{PhD}$ dissertation, University of Oxford. 2011.

[26] J. Ciston, Y. Wu, G. R. Odette, P. Hosemann, The structure of nanoscale precipitates and precipitate interfaces in an oxide dispersion strengthened steel, Micros. \& Microanal. 18 (2012) 760-761.

[27] N. J. Cunningham, Y. Wu, D. Klingensmith, G. R. Odette, On the remarkable thermal stability of nanostructured ferritic alloys, Mater. Sci. \& Eng. A. 613 (2014) 296-305.

[28] P. Miao, G. R. Odette, J. Gould, J. Bernath, R. Miller, M. Alinger, C. Zanis, The microstructure and strength properties of MA957 nanostructured ferritic alloy joints produced by friction stir and electro-spark deposition welding, J. Nuc. Mater. 367 (2007) 1197-1202.

[29] A. Etienne, N. J. Cunningham, Y. Wu, G. R. Odette, Effect of friction stir welding and post-weld annealing on nanostructured ferritic alloys, Mater. Sci. Technol. 27 (2011) 724-728.

[30] N. A. Bailey, E. Stergar, M. Toloczko, P. Hosemann, Atom probe tomography analysis of high dose MA957 at selected irradiation temperatures, J. Nuc. Mater. 459 (2015) 225-234.

[31] N. J. Cunningham, Y. Wu, G. R. Odette, D. T. Hoelzer, S. A. Maloy, Characterization of a larger best practice heat of 14YWT in annealed powder, HIP consolidated and extruded forms. Fusion Reactor Materials Semiannual Progress Report. DOE/ER-0313/54 (2013) 15-26. 
[32] U. Dahmen, R. Erni, V. Radmilovic, C. Ksielowski, M. Rossell, P. Denes, Background, status and future of the transmission electron aberration-corrected microscope project, Philos. Trans. Royal Soc. A. 367 (2009) 3795-3808.

[33] R. W. Gerchberg, W. O. Saxton, Practical algorithm for determination of phase from image and diffraction plane pictures, Opt. 35 (1972) 237-246.

[34] R. Kilaas, MacTempasX. 2014; http://www.totalresolution.com.

[35] A. Thust, M. Overwijk, W. Coene, M. Lentzen, Numerical correction of lens aberrations in phase-retrival HRTEM, Ultramicroscopy 64 (1996) 249-264.

[36] M. J. Hÿtch, E. Snoeck, R. Kilaas, Quantative measurement of displacement and strain fields from HREM micrographs, Ultramicroscopy 74 (1998) 131-146.

[37] N. V. Y. Scarlett, I. C. Madsen, Quantification of phases with partial or no known crystal structures, Powder Diffr. 21 (2006) 278-284.

[38] D. J. Sprouster, J. Sinsheimer, E. Dooryhee, S. K. Ghose, P. Wells, T. Stan, N. Almirall, G. R. Odette, L. E. Ecker, Structural characterization of highly irradiated reactor pressure vessel steels, Scr. Mater.113 (2016) 18-22.

[39] T. Okuda, M. Fujiwara, Dispersion behavior of oxide particles in mechanically alloyed ODS steel, Mater. Sci. Lett. 14 (1995) 1600-1603.

[40] H. Sakasegawa, M. Tamura, s. Ohtsuka, S. Ukai, H. Tanigawa, A. Kohyama, M. Fujiwara, Precipitation behavior of oxide particles in mechanically alloyed powder of oxide-dispersion-strengthened steels, J. Alloys and Compd. 452 (2008) 2-6.

[41] J. R. Rieken, I.E. Anderson, M. J. Kramer, G. R. Odette, E. Stergar, E. Haney, Reactive gas atomization processing for Fe-based ODS alloys, J. Nuc. Mater. 428 (2012) 65-75.

[42] A. J. London, B. K. Panigrahi, C. C. Tang, C. Murray, C. R. M. Grovenor, Glancing angle XRD analysis of particle stability under self-ion irradiation in oxide dispersion strengthened alloys, Scr. Mater. 110 (2016) 24-27.

[43] M. Ohnuma, J. Suzuki, S. Ohtsuka, S. -W. Kim, T. Kaito, M. Inoue, H. Kitazawa, A new method for the quantitative analysis of the scale and composition of nanosized oxide in 9Cr-ODS steel, Acta Mater. 57 (2009) 5571-5581.

[44] L. Barnard, G. R. Odette, I. Szlufarska, An ab initio study of Ti-Y-O nanocluster energetic in nanostructured ferritic alloys, Acta Mater. 60 (2012) 935-947.

[45] Y. Jiang, J. R. Smith, G. R. Odette, Formation of Y-Ti-O nanocluster in nanostructured ferritic alloys: A first-principles study, Phys. Rev. B 79 (2009) 064103.

[46] M. J. Alinger, B. D. Wirth, H. J. Lee, G. R. Odette, Lattice Monte Carlo simulation of nanocluster formation in nanostructured ferritic alloys, J. Nuc. Mater. 367 (2007) 153-159.

[47] Y. Jiang, J. R. Smith, G. R. Odette, Prediction of structure, electronic and elastic properties of $\mathrm{Y}_{2} \mathrm{Ti}_{2} \mathrm{O}_{7}$ and $\mathrm{Y}_{2} \mathrm{TiO}_{5}$, Acta Mater. 58 (2010) 1536-1543.

[48] L. Yang, Y. Jiang, G. R. Odette, T. Yamamoto, Z. Liu, Y. Liu, Trapping Helium in $\mathrm{Y}_{2} \mathrm{Ti}_{2} \mathrm{O}_{7}$ compared to matrix iron: A first principles study, J. Appl. Phys. 115 (2014) 143508.

[49] Y. Jin, Y. Jiang, L. Yang, G. Lan, G. R. Odette, T. Yamamoto, J. Shang, Y. Dang, First principles assessment of helium trapping in $\mathrm{Y}_{2} \mathrm{TiO}_{5}$ in nano-featured ferritic alloys, J. Appl. Phys. 116 (2014) 143501. 
[50] L. Yang, Y. Jiang, G. R. Odette, Nonstoichiometry and relative stabilities of $\mathrm{Y}_{2} \mathrm{TiO}_{7}$ polar surfaces: A density functional theory prediction, Acta Mater. 61 (2013) 7260-7270.

[51] L. Yang, Y. Jiang, Y. Wu, G. R. Odette, Z. Zhou, Z. Lu, The ferrite/oxide interface and helium management in nanostructured ferrritic alloys from the first principles, Acta Mater. 103 (2016) 474-482.

[52] L. Barnard, N. J. Cunningham, G. R. Odette, Thermodynamic and kinetic modeling of oxide precipitation in nanostructured ferritic alloys, Acta Mater. 91 (2015) 340-354. 


\section{Figures}
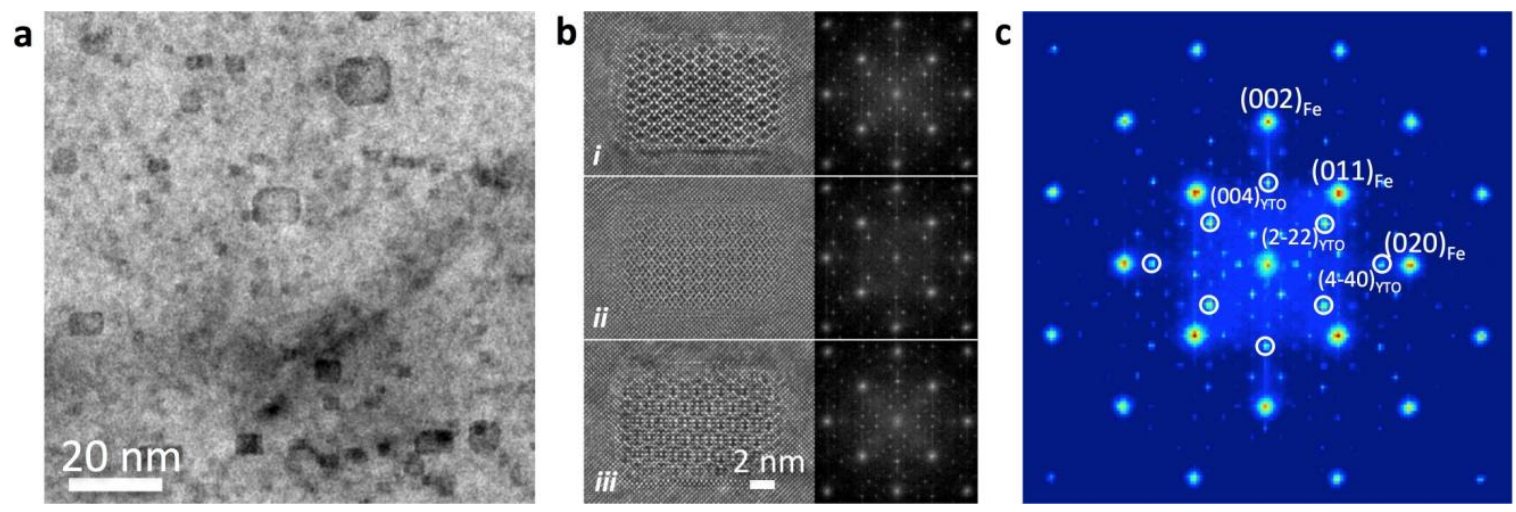

Figure 1. a, A bright field TEM image at low magnification of FSWA MA957 showing faceted, cuboidal oxide particles with a wide size distribution; b, representative HRTEM images from the focal series for a large NO and the corresponding power spectra for: i) under-; ii) near; and, iii) over-focus; and, c, the averaged FFT from all HRTEM images of the NO shown in Figure 1b, where the larger high-intensity reflections and their multiples are from the $\mathrm{Fe}-\mathrm{Cr}$ ferrite matrix $(\mathrm{Fe})$ while the white circles mark the major reflections of $\mathrm{Y}_{2} \mathrm{Ti}_{2} \mathrm{O}_{7}$ pyrochlore (YTO) with corresponding zone axes of [100] $]_{\mathrm{Fe}}$ and [110] $]_{\mathrm{YTO}}$. (Colorful on web) 


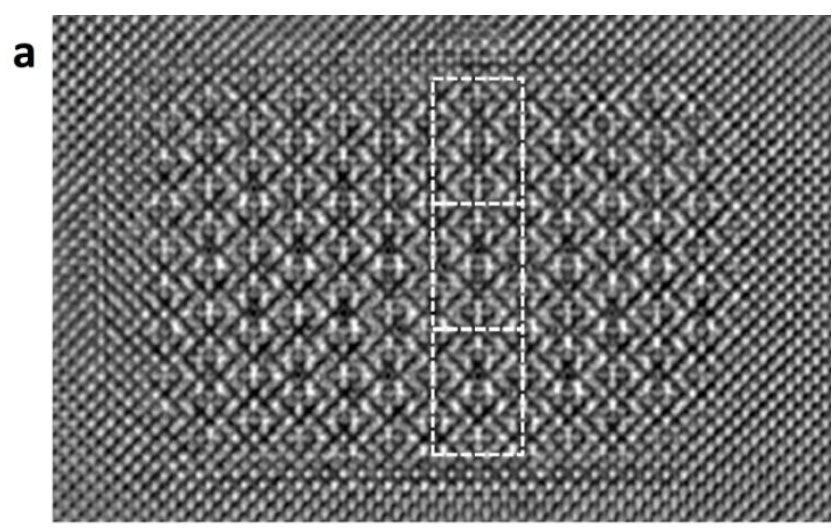

b

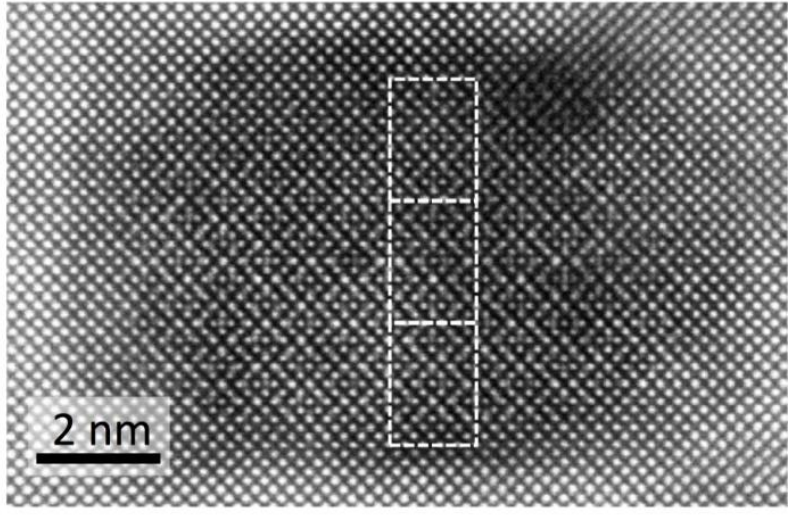

C


Figure 2. a, The phase of the reconstructed exit wave from of the focal series in Figure $1 \mathrm{~b} ; \mathbf{b}$, the corresponding HAADF STEM image where the dashed lines highlight the periodicity of the Moire pattern in the overlap region. c, magnified views of the periodic structure in the exit-wave reconstruction image, and $\mathbf{d}$, in the corresponding HAADF STEM image. The dashed lines show the periodic repeated pattern of $5 \times 7 \mathrm{Fe}$ unit cells, while the colored balls represent lateral positions of Y (green) and Ti (blue) columns relative to $\mathrm{Fe}$ (yellow) matrix.

(Colorful on web) 


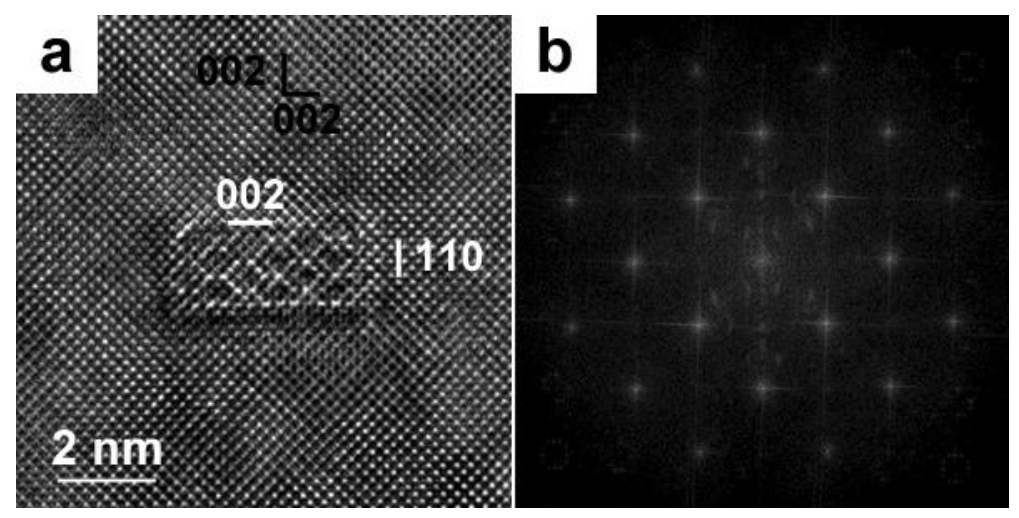

Figure 3. a. The HRTEM image of a small $1.8 \mathrm{~nm}$ by $3.3 \mathrm{~nm} \mathrm{NO}$; and, b. the corresponding FFT of this image. 


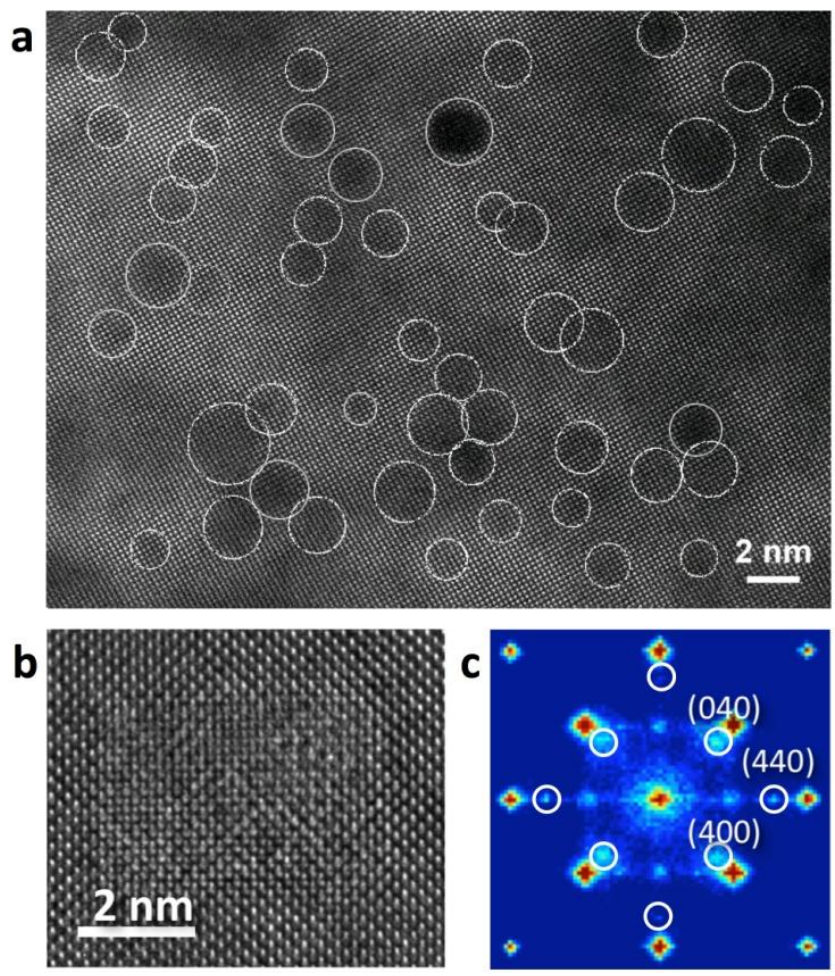

Figure 4. TEM images for AE MA957: a. a HAADF STEM overview image with circles marking some oxide particles - note circling all the NOs would make the figure far too cluttered; b. a HRTEM image of a slightly larger particle; and, c. an averaged FFT of all the images in the corresponding focal series. White circles mark the major reflections of $\mathrm{Y}_{2} \mathrm{Ti}_{2} \mathrm{O}_{7}$ pyrochlore (YTO). The corresponding zone axes are $[100]_{\mathrm{Fe}}$ and $[001]_{\mathrm{YTO}}$. (Colorful on web) 

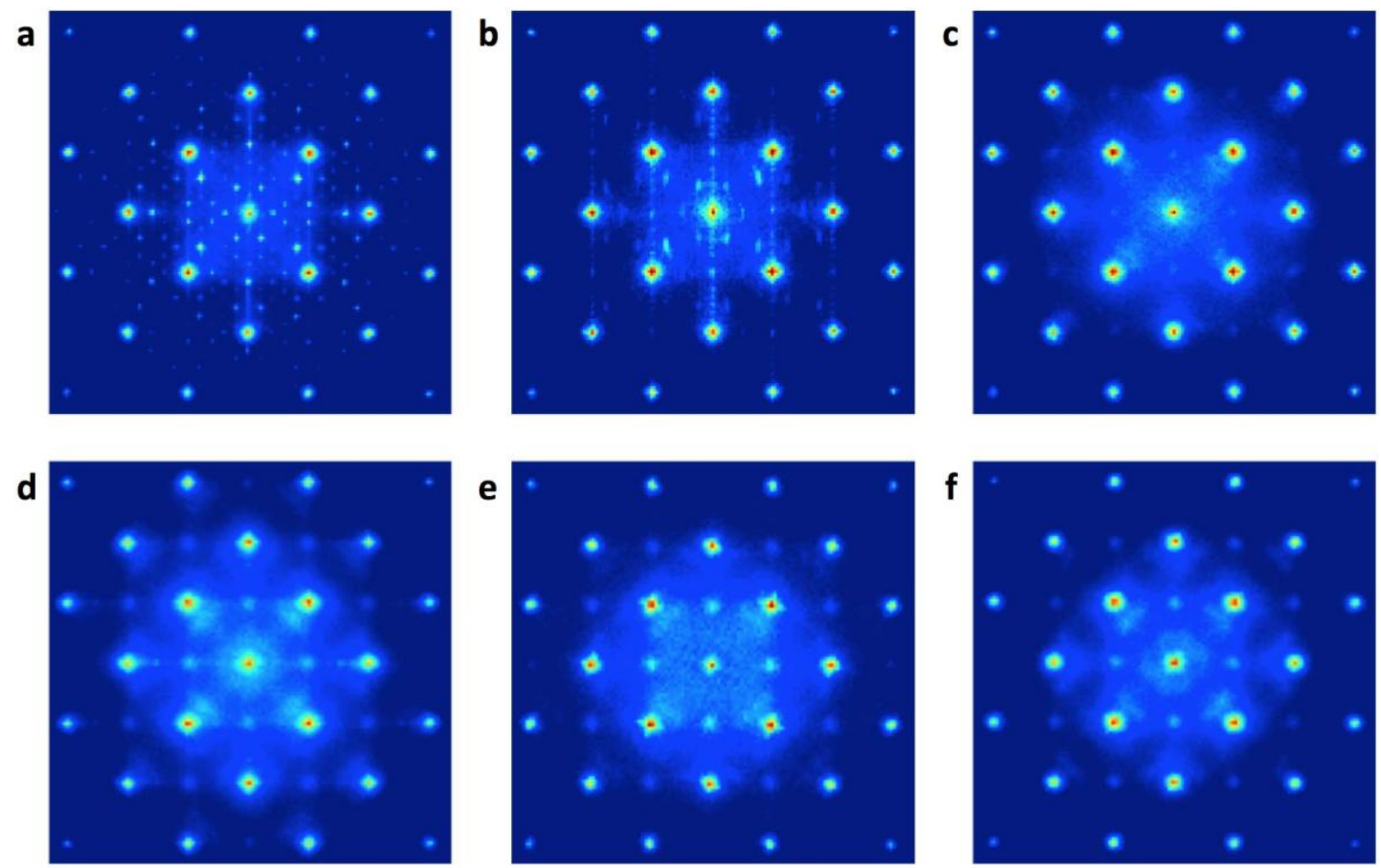

Figure 5. A summary of the averaged FFTs for all the NFA conditions studied. The top row is the FSWA MA957 for: $\mathbf{a}$, the large NO shown in Figure 1c; $\mathbf{b}$, a smaller 1.8x3.3 $\mathrm{nm}^{2} \mathrm{NO}$; and, $\mathbf{c}$, the area next to the small NO in b. The bottom row is for MA957 in the: d, AE MA957; e, IR MA957 conditions; and, f, NFA-1.

(Colorful on web) 
a

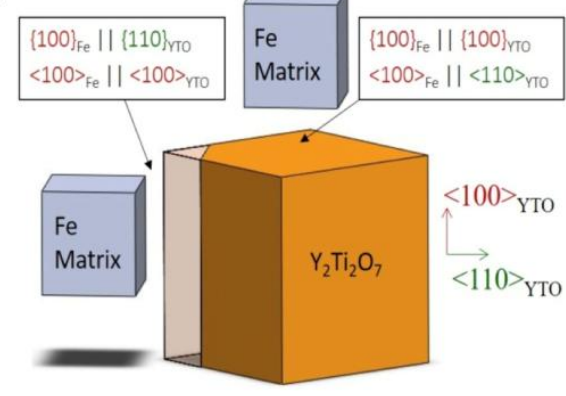

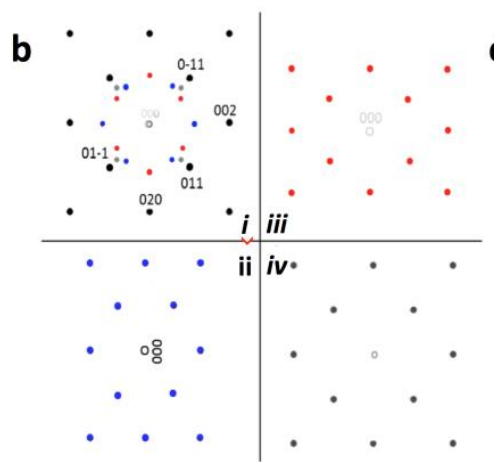

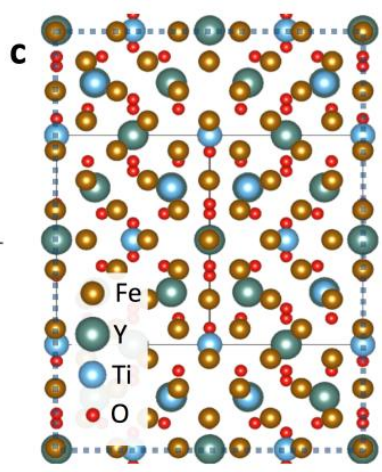

Figure 6. a, A schematic illustration of the cube on edge $\mathrm{OR}$ of $\mathrm{Y}_{2} \mathrm{Ti}_{2} \mathrm{O}_{7}$ pyrochlore (small cube) relative to the Fe matrix (large cube); b, i) all possible peak locations in the FFT assuming a cube-on-edge OR where ii) and iii) represent $\langle 110\rangle_{\text {YTO }}$ orientations rotated with respect to each other by $90^{\circ}$ and, iv) is $\langle 100\rangle_{\text {YTO }}$ rotated $45^{\circ}$ around the zone axis. c, The $5 \times 7$ array of Fe matrix unit cells in near-coincidence periodicity with the $\mathrm{Y}_{2} \mathrm{Ti}_{2} \mathrm{O}_{7}$ pyrochlore (cation) lattice.

(Colorful on web) 


\section{Tables}

Table1. NF size and \# density comparison for AE [A,B], FSWA [C], IR [D], and NFA-1 [E] conditions.

\begin{tabular}{cccccc}
\hline $\begin{array}{c}\text { NF } \\
\text { parameters }\end{array}$ & Technique & $\begin{array}{c}\text { MA957 } \\
(\text { AE) }\end{array}$ & $\begin{array}{c}\text { MA957 } \\
(\text { FSWA) }\end{array}$ & $\begin{array}{c}\text { MA957 } \\
(\text { IR })\end{array}$ & FCRD-NFA-1 \\
\hline Average & TEM & 2.9 & 3.0 & - & 2.1 \\
diameter & APT & 2.3 & 2.2 & 2.5 (Guinier $)$ & 2.0 \\
$(<\mathrm{d}>\mathrm{nm})$ & SANS & 2.7 & - & - & 3.0 \\
\hline & Average & 2.6 & 2.5 & 2.5 & 2.4 \\
\hline Number & TEM & 1.3 & 1.1 & - & 1.6 \\
density & APT & 3.2 & 0.9 & 5.1 & 6.9 \\
$\left(\mathrm{~N} 10^{23} \mathrm{~m}^{-3}\right)$ & SANS & 9.0 & - & - & 6.0 \\
\hline & Average & 4.5 & 2.1 & 5.1 & 4.8 \\
\hline
\end{tabular}

Table 2 Summary of d-spacings and plane angles measured in the indicated figures compared to bulk $\mathrm{Y}_{2} \mathrm{Ti}_{2} \mathrm{O}_{7}$

\begin{tabular}{lcccc}
\hline Figure & $\mathrm{d}(\AA)$ & $\mathrm{d}_{1}(2-22)$ & $\mathrm{d}_{2}(22-2)$ & $\alpha_{12}\left({ }^{\circ}\right)$ \\
\hline Fig. 5d & measured & $2.83 \pm 0.09$ & $2.83 \pm 0.09$ & $68.7 \pm 0.9$ \\
Fig. 5e & measured & $2.85 \pm 0.07$ & $2.85 \pm 0.07$ & $71.9 \pm 1.3$ \\
Fig. 5f & measured & $2.81 \pm 0.07$ & $2.80 \pm 0.07$ & $69.4 \pm 1.8$ \\
& $\mathrm{Y}_{2} \mathrm{Ti}_{2} \mathrm{O}_{7}$ & 2.90 & 2.90 & 70.5 \\
Fig. 4c & $\mathrm{d}(\AA)$ & $\mathrm{d}_{1}(400)$ & $\mathrm{d}_{2}(040)$ & $\alpha_{12}\left({ }^{\circ}\right)$ \\
& measured & $2.60 \pm 0.08$ & $2.60 \pm 0.08$ & $90.6 \pm 1.8$ \\
& $\mathrm{Y}_{2} \mathrm{Ti}_{2} \mathrm{O}_{7}$ & 2.52 & 2.52 & 90 \\
\hline
\end{tabular}


a

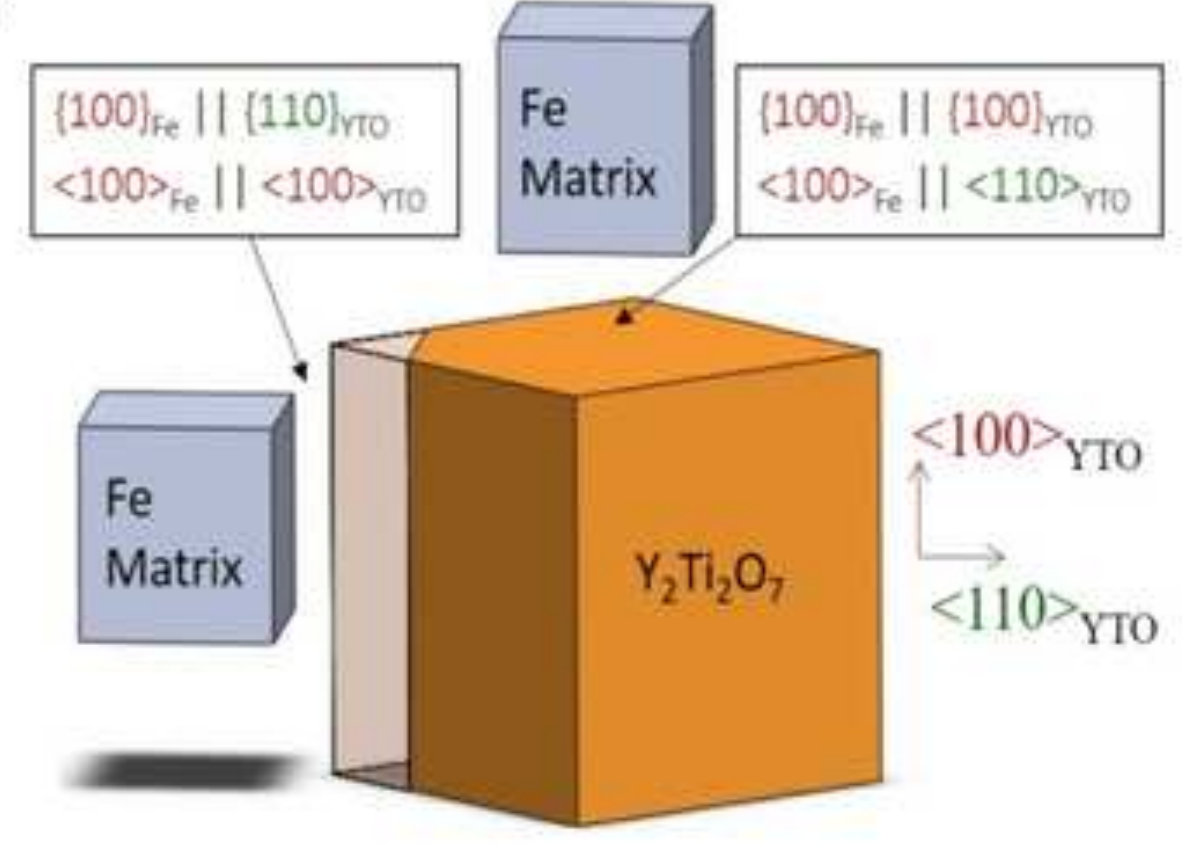

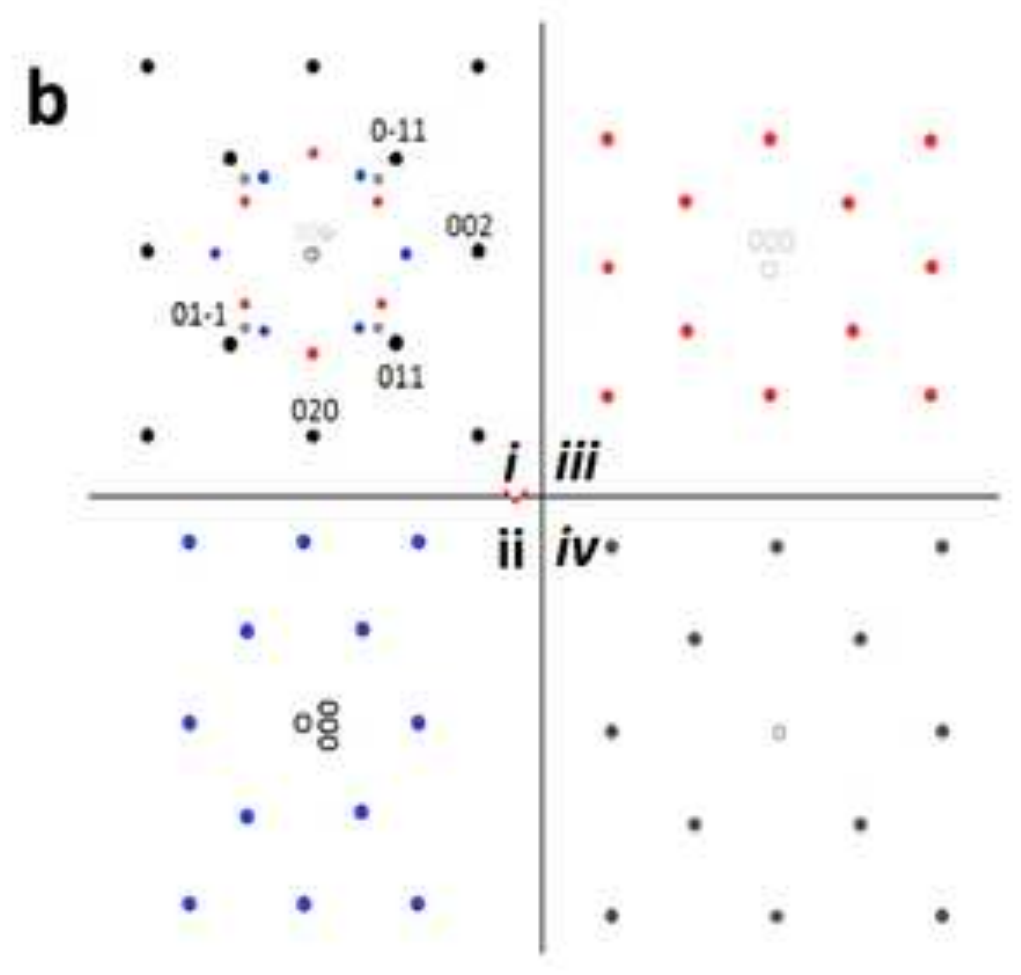

